\title{
Improving the Performance of Multistage Interconnection Networks under Nonuniform Traffic Pattern on Shorter Cycles
}

\author{
Hyunseung $\mathrm{Choo}^{1}$ and Youngsong Mun ${ }^{2}$ \\ ${ }^{1}$ School of Information and Communication Engineering \\ Sungkyunkwan University, Suwon, Korea \\ choo@skku. edu \\ ${ }^{2}$ School of Computing, Soongsil University, Seoul, Korea \\ mun@computing.ssu.ac.kr
}

\begin{abstract}
Multistage interconnection networks (MINs) have been recently identified as an efficient interconnection network for a switching fabric of communication structures such as gigabit ethernet switch, terabit router, and ATM switch. Even though there have been a number of studies about modeling MINs in the literature, almost all of them are for trends MINs under uniform traffic which dose not reflect the realistic. In this paper, we propose an analytical model to evaluate the performance of ATM switches based on MINs with the small clock cycle (SCC) scheme under nonuniform traffic. Here MINs of 6 and 10 stages with built-in buffer modules holding single or multiple cells are considered for the evaluation. Comprehensive computer simulation results present that the proposed model is effective for predicting the performance of ATM switches under the realistic nonuniform traffic. It also shows that the detrimental effect on the hot spot traffic which is typical in the Internet turns out to be more significant as the switch size increases.
\end{abstract}

\section{Introduction}

Multistage interconnection network (MIN) constructed by switching elements (SEs) with simple connections in multiple stages has been recognized as an efficient interconnection structure for super computer [1] and faster network devices [2]. Its regularity and modularity make MIN very appropriate for very large scale integration. It also has the self-routing capability, which is important for fast input to output connection. MINs thus have been considered for the switching fabric of new communication structures such as gigabit Ethernet switch [3], terabit router, and ATM switch.

ATM [4] is a high-speed connection-oriented packet switching technique which employs short fixed-length packets called cells. It allows much higher rate switching than typical packet switching due to its functional simplification. While numerous switching systems for ATM have been proposed, MIN is identified to be very effective for integrated service digital network (ISDN) and also it is expected to be an 
outstanding candidate for the future networks supporting broadband services such as multimedia data and graphic oriented applications. Understanding and predicting the performance of the switches are very important in designing correct switch-based communication systems. There have been a number of studies investigating the performance of MINs in the literature [5-7]. However, almost all of these previous works are for studying the MINs under the uniform traffic pattern. Although the performance under uniform traffic is interesting to note, it does not provide the actual data on the real performance of MINs. The nonuniform traffic reflects the realistic traffic pattern of currently deployed integrated service networks where a wide range of bandwidths needs to be accommodated. Therefore, the performance of the MINs under nonuniform traffic must be studied for obtaining an efficient switch-based system. Even though there are few number of models investigated on nonuniform traffic patterns [6-7], they are not good enough on the performance of the models and even not reasonably verified.

In this paper, we propose an analytical modeling method to evaluate the performance of ATM switches based on MINs with small clock cycle (SCC) scheme under the nonuniform traffic. It is mainly achieved by properly implementing the nonuniform dispatch probability in modeling the operation for each switching element. To evaluate the accuracy of the proposed model, comprehensive computer simulation is performed on comparison purpose for two performance measures - throughput and delay. MINs with 6 and 10 stages with built-in buffer modules holding single or multiple cells are considered for the evaluation. As a typical nonuniform traffic pattern, hot spot traffic of 3.5\% and 7\% are investigated the comparison of the simulation data with the data obtained from the analytical model shows that the proposed method is very effective for predicting the performance of ATM switches under the realistic nonuniform traffic. The detrimental effect on the hot spot traffic on the network performance turns out to get more significant as the switch some increases.

\section{The Proposed Model}

The SCC consists of two phases in our models. In the first phase, the sending buffer modules check the empty space availability of the receiving buffer modules. Based on the availability (routing information) of the succeeding stages, each sending buffer module sends a packet to its destination or enters into the blocked state in the second phase. Other assumptions used in our model are as follows.

- $2 \times 2$ SEs with the buffer modules of size $m$ are used, where the buffer modules are located at the input port; All packets have the same size (like in ATM); The probability that a packet arrives at each network input is the same for all inputs. Each inlet generates requests identically with a certain probability which are nonuniformly distributed over all outlets; Two buffer modules in a same $2 \times 2$ SE are statistically independent; Each packet has an equal probability to win the contention; A blocked packet is resubmitted to the original destination. 
The number of possible states of a buffer module of size $m$ is $2 \mathrm{~m}$ such that State- 0 , State $-\mathrm{n}_{\mathrm{k}}(1 \leq \mathrm{k} \leq \mathrm{m}-1)$, and State $-\mathrm{b}_{\mathrm{k}}(1 \leq \mathrm{k} \leq \mathrm{m})$. The state transition diagram of this model is shown in figure 1. Note that State- $\mathrm{n}_{\mathrm{m}}$ does not exist. This is because if a buffer module is full, then it cannot receive a packet. If the packet at the head left the module, it would be in State- $\mathrm{n}_{(\mathrm{m}-1)^{*}}$. Otherwise, in State- $\mathrm{b}_{\mathrm{m}}$. Note also that the state of a buffer module depends only on the oldest packet (the packet at the head of the buffer module) whether it has participated in a contention or not. The buffers are assumed to be serviced on a first-come-first-served (FCFS) manner.

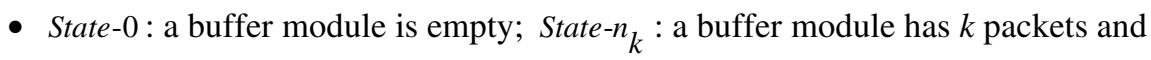
the head packet moved into the current position in the previous network cycle; State- $b_{k}$ : a buffer module has $k$ packets and the head packet could not move forward due to the lack of the empty space of its destined buffer module in the previous network cycle.

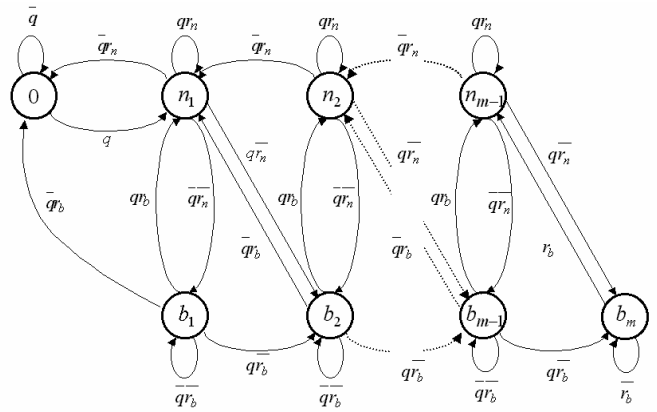

Fig. 1. The state transition diagram of the proposed model

The following variables are defined to develop our analytical model. Here $Q(i j)$ denotes a $j$-th buffer module in stage $i$ and two ports in the same SE are called as a conjugate port each other. We denote $Q\left(i j^{c}\right)$ as the conjugate buffer module of $Q(i j)$. Also $t_{b}$ represents the time instance when a network cycle begins, while $t_{d}$ represents the duration of a network cycle.

- $m$ : number of buffers in a buffer module; $n$ : number of switching stages. There are $n=\log _{2} N$ stages for $N \times N$ MINs.

- $P_{0}(i j, t)$ : probability that $Q(i j)$ is empty at $t_{b} ; \overline{P(i j, t)}$ : probability that $Q(i j)$ is not full at $t_{b} ; P_{n_{k}}(i j, t) / P_{n_{k}}\left(i j^{c}, t\right)$ : probability that $Q(i j) / Q\left(i j^{c}\right)$ is in State - $n_{k}$ at $t_{b}$, where $1 \leq k \leq m-1 ; P_{b_{k}}(i j, t) / P_{b_{k}}\left(i j^{c}, t\right)$ : probability that $Q(i j) / Q\left(i j^{c}\right)$ is in State $-b_{k}$ at $t_{b}$, where $1 \leq k \leq m ; P_{b}^{u}(i j, t) / P_{b}^{l}(i j, t)$ : probability that a head 
packet in $Q(i j)$ is a blocked one and it is destined to the upper / lower output port at $t_{b} ; S P_{n}(i j, t): \sum_{k=1}^{m-1} P_{n_{k}}(i j, t)$, and $S P_{b}(i j, t): \sum_{k=1}^{m} P_{b_{k}}(i j, t)$.

- $q(i j, t)$ : probability that a packet is ready to come to the buffer module $Q(i j)$ during $t_{d}$.

- $r(i j) / r_{x}(i j, t)$ : probability that a normal/blocked head packet in $Q(i j)$ is destined to the upper output port; $r_{n}(i j, t) / r_{b}(i j, t)$ : probability that a normal/blocked packet at the head of $Q(i j)$ is able to move forward during $t_{d}$;

- $r_{n}^{u}(i j, t) / r_{n}^{l}(i j, t)$ : probability that a normal packet at the head of $Q(i j)$ can get to the upper / lower output port during $t_{d} ; \quad r_{n n}^{u}(i j, t) / r_{n n}^{l}(i j, t)$ : probability that a normal packet at the head of $Q(i j)$ can get to the upper / lower output port during $t_{d}$ by considering $Q\left(i j^{c}\right)$ in either State- $n_{k}$ or State- $b_{k}$. If $Q\left(i j^{c}\right)$ is in State $-b_{k}$, it is assumed that the blocked packet is destined to the lower / upper port (so no contention is necessary); $r_{n b}^{u}(i j, t) / r_{n b}^{l}(i j, t)$ : probability that a normal packet at the head of $Q(i j)$ is able to get to the upper / lower output port during $t_{d}$ by winning the contention with a blocked packet at the head of $Q\left(i j^{c}\right)$;

- $r_{b}^{u}(i j, t) / r_{b}^{l}(i j, t)$ : probability that a blocked packet at the head of $Q(i j)$ can get to the upper / lower output port during $t_{d} ; r_{b n}^{u}(i j, t) / r_{b n}^{l}(i j, t)$ : probability that a blocked packet at the head of $Q(i j)$ is able to move forward to the upper / lower output port during $t_{d}$. Here it is assumed that $Q\left(i j^{c}\right)$ is empty or in the State $-n_{k} ; r_{b b}^{u}(i j, t) / r_{b b}^{l}(i j, t)$ : probability that a blocked packet at the head of $Q(i j)$ is able to move forward to the upper / lower output port during $t_{d}$. Here it is assumed that $Q\left(i j^{c}\right)$ also has a blocked packet.

- $P^{n a}(i j, t) / P^{b a}(i j, t) / P^{b b a}(i j, t)$ : probability that a buffer space in $Q(i j)$ is available (ready to accept packets) during $t_{d}$, given that no blocked packet / only one blocked packet / two blocked packets in the previous stage is destined to that buffer module.

- $X_{n}^{u}(i j, t) / X_{n}^{l}(i j, t)$ : probability that a normal packet destined to the upper / lower output port is blocked during $t_{d} ; X_{b}^{u}(i j, t) / X_{b}^{l}(i j, t)$ : probability that a blocked packet destined to the upper / lower output port is blocked during $t_{d}$.

- $T(i j, t)$ : probability that an input port of $Q(i j)$ receives a packet. 


\subsection{Calculations for the Required Measures}

[Obtaining $r_{n}(i j, t)$ ] A normal packet in an SE is always able to get to its desired output port when the other buffer is empty or it is destined to a different port from that of the normal packet in the other buffer. When two normal packets compete, each packet has the equal probability to win the contention. The probability that a normal packet in $Q(i j)$ does not compete with a blocked packet in the other buffer is $r(i j)\left\{1-r_{x}\left(i j^{c}, t\right)\right\}$ or $\{1-r(i j)\} r_{x}\left(i j^{c}, t\right)$. Therefore, probabilities $r_{n n}^{u}(i j, t)$ is as follows and $r_{n n}^{l}(i j, t)$ is obtained similarly.

$$
\begin{aligned}
r_{n n}^{u}(i j, t) & =r(i j) P_{0}\left(i j^{c}, t\right)+\left[0.5 r(i j) r\left(i j^{c}\right)+r(i j)\left\{1-r\left(i j^{c}\right)\right\}\right] S P_{n}\left(i j^{c}, t\right) \\
& +r(i j)\left\{1-r_{x}\left(i j^{c}, t\right)\right\} S P_{b}\left(i j^{c}, t\right)
\end{aligned}
$$

$r_{n b}^{u}(i j, t)$ and $r_{n b}^{l}(i j, t)$ are probabilities that a packet has the same destination as the blocked packet in the other buffer and it wins the contention. Thus they are as follows:

$$
r_{n b}^{u}(i j, t)=0.5 r(i j) r_{x}\left(i j^{c}, t\right) S P_{b}\left(i j^{c}, t\right)
$$

The probability that a buffer module is not full $(\overline{P(i j, t)})$ is simply

$$
\overline{P(i j, t)}=1-P_{b_{m}}(i j, t) \text {. }
$$

For $P^{b a}(i j, t)$, the originating buffer module of a packet is in State - $b_{k}$. If $Q(i j)$ received a packet in the previous network cycle with probability of $T(i j, t-1)$, it should not have been full. To make a space to accept a packet in network cycle $t$, a packet must have been moved if it had only one available space. If it did not receive a packet in the previous network cycle with the probability of $1-T(i j, t-1)$, it must have been full. To make a space to accept a packet in network cycle $t$, a packet must have been moved from the full buffer. Thus

$$
P^{b a}(i j, t)=T(i j, t-1) \times A+\{1-T(i j, t-1)\} \frac{P_{b_{m}}(i j, t-1) r_{b}(i j, t-1)}{P_{b_{m}}(i j, t-1)} .
$$

where

$$
A=\frac{P_{0}(i j, t-1)+\sum_{k=1}^{m-2} P_{n_{k}}(i j, t-1)+\sum_{k=1}^{m-2} P_{b_{k}}(i j, t-1)+P_{n_{m-1}}(i j, t-1) r_{n}(i j, t-1)+P_{b_{m-1}}(i j, t-1) r_{b}(i j, t-1)}{P_{0}(i j, t-1)+\sum_{k=1}^{m-1} P_{n_{k}}(i j, t-1)+\sum_{k=1}^{m-1} P_{b_{k}}(i j, t-1)}
$$

$P^{n a}(i j, t)$ implies that the destined buffer can be in any state because no blocked packet is destined to it. If it received a packet in the previous network cycle with probability of $T(i j, t-1)$, it should have not been full. To make a space to accept a packet in network cycle $t$, a packet must have been moved if it had only one available space. If it did not receive a packet in the previous network cycle with probability of 
$1-T(i j, t-1)$, it could be in any state. To make a space to accept a packet in the network cycle $t$, a packet must have been if the buffer was full. $P^{n a}(i j, t)$ is as follows.

$$
P^{n a}(i j, t)=T(i j, t-1) \times A+\{1-T(i j, t-1)\} \times B .
$$

where $B=\frac{P_{0}(i j, t-1)+\sum_{k=1}^{m-1} P_{n_{k}}(i j, t-1)+\sum_{k=1}^{m-1} P_{b_{k}}(i j, t-1)+P_{b_{m-1}}(i j, t-1) r_{b}(i j, t-1)}{P_{0}(i j, t-1)+\sum_{k=1}^{m-1} P_{n_{k}}(i j, t-1)+\sum_{k=1}^{m} P_{b_{k}}(i j, t-1)}$.

For a packet to move to the succeeding stage, it should be able to get to the desired output port as well as the destined buffer module should be available. Thus $r_{n}^{u}(i j, t)$ is

as follows and $r_{n}^{l}(i j, t)$ is obtained similarly.

$$
r_{n}^{u}(i j, t)=r_{n n}^{u}(i j, t) P^{n a}((i+1) e, t)+r_{n b}^{u}(i j, t) P^{b a}((i+1) e, t),
$$

So $r_{n}(i j, t)$ is

$$
r_{n}(i j, t)=r_{n}^{u}(i j, t)+r_{n}^{l}(i j, t) .
$$

We can calculate $r_{b}(i j, t)$ using the similar method and we skip the detail due to the space constraint.

[Calculating $X_{n}^{u}(i j, t), X_{b}^{u}(i j, t), X_{n}^{l}(i j, t), X_{b}^{l}(i j, t), r_{x}(i j, t), T(i j, t)$ and $q(i j, t)$ ] $X_{n}^{u}(i j, t)$ is the probability that a normal packet destined to the upper output port is blocked. Therefore $X_{n}^{u}(i j, t)$ is

$$
\begin{aligned}
X_{n}^{u}(i j, t) & =r_{n n}^{u}(i j, t)\left\{1-P^{n a}((i+1) e, t)\right\}+r_{n b}^{u}(i j, t)\left\{1-P^{b a}((i+1) e, t)\right\} \\
& +0.5 r(i j) r\left(i j^{c}\right) S P_{n}\left(i j^{c}, t\right)+0.5 r(i j) r_{x}\left(i j^{c}, t\right) S P_{b}\left(i j^{c}, t\right)
\end{aligned} .
$$

The first two terms are probabilities for the destination unavailable. The last two terms are for the contention losing. $X_{b}^{u}(i j, t)$ is the probability that a blocked packet destined to the upper output port is blocked again. We can calculate this probability easily by the approach employed in $X_{n}^{u}(i j, t)$.

$$
\begin{aligned}
X_{b}^{u}(i j, t) & =r_{b n}^{u}(i j, t)\left\{1-P^{b a}((i+1) e, t)\right\}+r_{b b}^{u}(i j, t)\left\{1-P^{b b a}((i+1) e, t)\right\} \\
& +0.5 r_{x}(i j) r\left(i j^{c}\right) S P_{n}\left(i j^{c}, t\right)+0.5 r_{x}(i j) r_{x}\left(i j^{c}, t\right) S P_{b}\left(i j^{c}, t\right)
\end{aligned}
$$

$X_{n}^{l}(i j, t)$ and $X_{b}^{l}(i j, t)$ are obtained similarly.

Also $r_{x}(i j, t)$, which is the probability that a blocked head packet is destined to the upper output port is calculated as follows.

$$
r_{x}(i j, t)=\frac{P_{b}^{u}(i j, t-1)}{P_{b}^{u}(i j, t-1)+P_{b}^{l}(i j, t-1)} \quad\left(P_{b}^{u}(i j, t-1)+P_{b}^{l}(i j, t-1) \neq 0\right)
$$


Here $P_{b}^{u}(i j, t)$ and $P_{b}^{l}(i j, t)$ are calculated as follows.

$$
\begin{aligned}
& P_{b}^{u}(i j, t)=X_{n}^{u}(i j, t) S P_{n}(i j, t)+X_{b}^{u}(i j, t) S P_{b}(i j, t), \\
& P_{b}^{l}(i j, t)=X_{n}^{l}(i j, t) S P_{n}(i j, t)+X_{b}^{l}(i j, t) S P_{b}(i j, t) .
\end{aligned}
$$

If the buffer module $Q(i j)$ for Stage-i $(2 \leq i \leq n)$ is connected into the upper output ports of the previous stage $\left(Q((i-1) g)\right.$ and $\left.Q\left((i-1) g^{c}\right)\right)$, the throughput of $Q(i j)$ is as follows.

$$
\begin{aligned}
T(i j, t)= & S P_{n}((i-1) g, t) r_{n}^{u}((i-1) g, t)+S P_{n}\left((i-1) g^{c}, t\right) r_{n}^{u}\left((i-1) g^{c}, t\right) \\
& +S P_{b}((i-1) g, t) r_{b}^{u}((i-1) g, t)+P_{b}\left((i-1) g^{c}, t\right) r_{b}^{u}\left((i-1) g^{c}, t\right)
\end{aligned} .
$$

The buffer modules which are connected to lower output ports of the previous stage are obtained similarly. $T(i j, t)(1 \leq i \leq n)$ also has the following relation with $T(i j, t)$.

$$
T(i j, t)=q(i j, t)\left[\overline{P(i j, t)}+P_{n_{m}}(i j, t) r_{n}(i j, t)+P_{b_{m}}(i j, t) r_{b}(i j, t)\right]
$$

Finally, $q(i j, t)(2 \leq i \leq n)$ is obtained.

$$
q(i j, t)=\frac{T(i j, t)}{\overline{P(i j, t)}+P_{n_{m}}(i j, t) r_{n}(i j, t)+P_{b_{m}}(i j, t) r_{b}(i j, t)}
$$

We need to separately consider the first and last stages because they have different conditions from other stages inside.

\subsection{Calculating $r(i j)$, Throughput, and Delay}

$r(i j)$ is calculated by using the transformation method studied in [8]. It is a mapping scheme that transforms the given referencing pattern into a set of $r(i j)$ 's which reflects the steady state traffic flow in the network. For a steady state referencing pattern, we represent it in terms of destination accessing probabilities $A_{j}$, the probability that a new packet generated by an inlet chooses the output port $j$ as its destination. Then $r(i j)$ can be represented as the conditional probability that the sum of $A_{j}$ 's which are connected by the upper output port of $Q(i j)$ under the given condition that the sum of $A_{j}$ 's of all possible destined output ports which are connected by the upper or lower output ports of $Q(i j)$. For example, $r(i j)$ 's in three-stage MIN are as follows.

For the last stage: $r(31)=r(32)=\frac{A_{1}}{A_{1}+A_{2}}, r(33)=r(34)=\frac{A_{3}}{A_{3}+A_{4}}$,

For the second stage: $r(21)=r(22)=r(25)=r(26)=\frac{A_{1}+A_{2}}{A_{1}+A_{2}+A_{3}+A_{4}}$,

For the first stage, all $r(1 j)(1 \leq j \leq N)$ are same: 


$$
r(11)=r(12)=\ldots=r(18)=\frac{A_{1}+A_{2}+A_{3}+A_{4}}{A_{1}+A_{2}+A_{3}+A_{4}+A_{5}+A_{6}+A_{7}+A_{8}}=A_{1}+A_{2}+A_{3}+A_{4}
$$

Normalized throughput of a MIN is defined to be the throughput of an output port of the last stage. If a port $j$ is the upper output port of an SE, the normalized throughput in this port is as follows. If a port $j$ is the lower output port of an SE, then it obtained similarly.

$$
\begin{aligned}
\operatorname{TNET}(j, t) & =S P_{n}(n g, t) r_{n}^{u}(n g, t)+S P_{n}\left(n g^{c}, t\right) r_{n}^{u}\left(n g^{c}, t\right) \\
& +S P_{b}(n g, t) r_{b}^{u}(n g, t)+S P_{b}\left(n g^{c}, t\right) r_{b}^{u}\left(n g^{c}, t\right)
\end{aligned}
$$

The delay occurred for a packet at the buffer module $Q(i j)$ in the steady state is calculated by using Little's formula.

$$
D(i j)=\lim _{t \rightarrow \infty} \frac{\sum_{k=1}^{m-1} k\left\{P_{n_{k}}(i j, t)+P_{b_{k}}(i j, t)\right\}+m P_{b_{m}}(i j, t)}{T(i j, t)}
$$

As each port delay is different, the weight of it should be considered for the mean delay. Hence the mean delay is

$$
D=\sum_{j=1}^{N} w_{j} D(j)
$$

Here $w_{j}$ - the weight of port $\mathrm{j}$ for the mean delay - is obtained by the rate of the normalized throughput of that port as follows.

$$
w_{j}=\lim _{t \rightarrow \infty} \frac{\operatorname{TNET}(j)}{\sum_{k=1}^{N} \operatorname{TNET}(k, t)}
$$

\section{Performance Verification of the Proposed Model}

Correctness of our model in terms of the network throughput and delay is verified by comparing them with the data obtained from computer simulation for various buffer sizes and traffic conditions. For the simulation, 95\% confidence interval is used and following approaches are employed for the computer simulation.

- Each inlet generates requests at the rate of the offered input traffic load; The destination of each packet follows the given nonuniform traffic pattern - hot spot traffic pattern. Here each inlet makes a fraction $h$ of their requests to a hot spot port, while the remaining $(1-h)$ of their requests are distributed uniformly over all output ports including the hot spot port. Assume that usually the output port 1 is the hot spot port. If there is a contention between the packets in an SE, it is resolved randomly; The buffer operation is based on the FCFS principle. 
Figure 2 shows the mean throughput and delay comparison of 6-stage single buffered MIN with the $7 \%$ hot spot traffic pattern using computer simulation. It plots the throughput and delay data for MIN's containing buffer modules with capacity to hold one cell (single buffered) of six stages $(64 \times 64)$ respectively. The offered traffic load varies form 0.1 to 1 , and data are collected from the computer simulation. Here each simulation data is obtained by averaging 10 runs. In each run, 1,000,000 iterations are taken to collect reliable data, and the variations in the last 100,000 iterations are less than $0.1 \%$. Figure 3 shows that the throughput comparison of the hot spot port and other ports in this case. The throughput of the hot spot port is much more than other ports since heavy traffics are concentrated on the hot spot port. Figures 4 and 5 show that comparison results of the more buffered MIN. In case of the uniform traffic pattern, more buffers can increase the performance of MINs (15 20\%) in terms of throughput. In our model, the throughput of the multi-buffered MIN with the $3.5 \%$ hot spot traffic pattern is more increased (50\%). But that with the $7 \%$ hot spot traffic pattern is not increased since severe blocking caused by the concentration of the traffic decreases the performance.
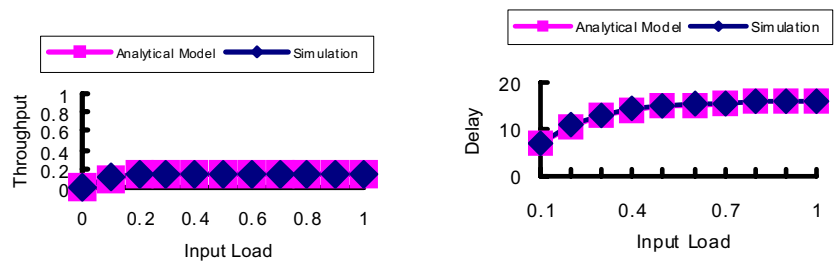

Fig. 2. Throughput and mean delay comparison of 6-stage single-buffered MIN with $7 \%$ the hot spot traffic pattern

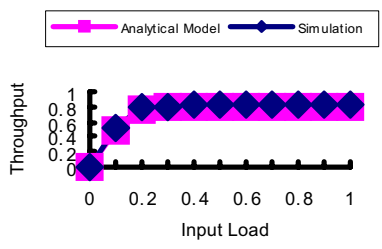

a) hot spot port

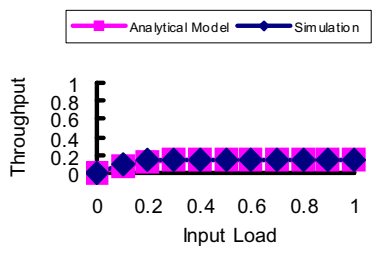

b) other ports

Fig. 3. Throughput comparison of 6-stage single-buffered MIN with $7 \%$ the hot spot traffic pattern

These figures show that our models are exactly accurate in terms of the throughput under the practical size MIN. Delay is also very accurate in single buffered MIN but the accuracy of it is somewhat decreased in case of the multi-buffered MIN. For more sized MIN, the throughput and delay have the stable value from the time that the input load is very low (0.1) since heavy traffics are concentrated form a lot of (1024) input ports. The practical performance is very low (about 0.03 ) which means that switching structures are not useful when their size is very large with nonuniform traffics patterns. This phenomenon is observed similarly when we compare the multi-buffered cases. 

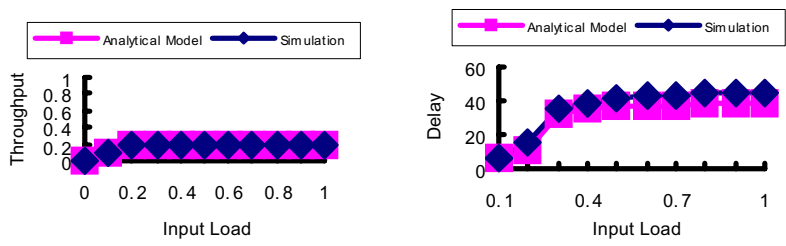

Fig. 4. Throughput and mean delay comparison of 6-stage 4-buffered MIN with 7\% the hot spot traffic pattern

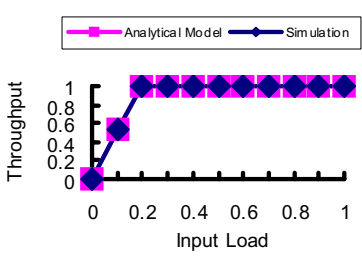

a) hot spot port

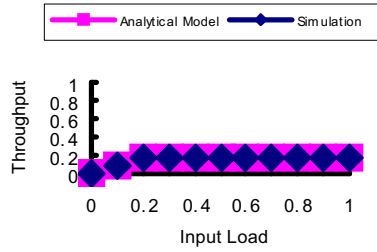

b) other ports

Fig. 5. Throughput comparison of 6-stage 4-buffered MIN with $7 \%$ the hot spot traffic pattern

\section{Conclusion}

This paper has proposed an analytical modeling method for the performance evaluation of MINs with $2 \times 2$ switching elements using small clock cycles (SCC) under nonuniform traffic. The effectiveness of the proposed model was verified by computer simulation for various practical MINs; $6 \times 6$ and $10 \times 10$ switches, single and 4 buffered MIN with $3.5 \%$ and $7 \%$ hot spot traffic. According to the results, the proposed model is very accurate in terms of throughput and delay. The detrimental effect of hot spot traffic on the network performance turns out to get more significant as the switch size increases. Therefore hot spot traffic needs to be avoided as much as possible for especially relatively large size switches.

\section{References}

1. C. L. Wu and T. Y. Feng, " On a class of Multistage Interconnection Networks," IEEE Trans. on Computers, Vol. C-29, pp. 694-702, August 1980.

2. J.S. Turner, "Design of and integrated services packet networks," Ninth Data Commun. Symp., in ACM SigComm Comput. Commun. Rev., vol. 15, pp. 124-133, Sept. 1985.

3. Muh-rong Yang and GnoKou Ma, "BATMAN: A New Architectural Design of a Very Large Next Generation Gigabit Switch," IEEE International Conference on Communications, Vol.2/3, pp. 740-744, May 1997.

4. H. Rudin, "The ATM-Asynchronous Transfer Mode," Computer Networks and ISDN Systems, Vol. 24, pp. 277-278, 1992. 
5. Y.C. Jenq, "Performance analysis of a packet switch based on single buffered Banyan network,” IEEE J. Select. Areas Commun, vol. SAC-3, pp. 1014-1021, Dec. 1983.

6. H. Kim and A. Leon-Garcia, "Performance of Buffered Banyan Networks Under Nonuniform Traffic Patterns," IEEE Transaction on Communications, Vol. 38, No. 5, May 1990.

7. T. Lin and L. Kleinrock, "Performance Analysis of Finite-Buffered Multistage Interconnection Networks with a General Traffic Pattern", ACM SIGMETRICS Conference on Measurement and Modeling of Computer Systems, San Diego, CA, pp. 68-78, May 21-24, 1991.

8. H.Y. Youn and H. Choo, "Performance Enhancement of Multistage Interconnection Networks with Unit Step Buffering,” IEEE Trans. on Commun. Vol. 47, No. 4, April 1999. 